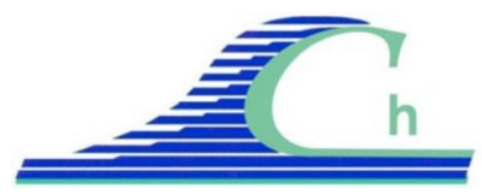

XII ${ }^{\text {èmes }}$ Journées Nationales Génie Côtier - Génie Civil

Cherbourg, 12-14 juin 2012

DOI:10.5150/jngcgc.2012.109-B @ Editions Paralia CFL

disponible en ligne - http://www.paralia.fr - available online

\title{
Méthodologie SETARMS de caractérisation et de traitement des sédiments de dragage marin
}

\author{
Mohamed BOUTOUIL ${ }^{1,2}$, Nor Edine ABRIAK ${ }^{3}$, \\ Lucile SAUSSAYE $^{1,2}$, Mahfoud BENZARZOUR ${ }^{3}$
}

1. ESITC Caen, ESITC Caen, Ecole Supérieure d'Ingénieurs des Travaux de la Construction de Caen, F-14610 Epron, France.

dir.recherche@esitc-caen.fr ; ecr.geotech@esitc-caen.fr

2. Université de Caen Basse-Normandie, Unité de Recherche Aliments Bioprocédés Toxicologie Environnements (UR ABTE) EA 4651, F-14032 Caen, France.

3. EMD, Département Génie Civil \& Environnemental de l'Ecole des Mines de Douai, F-59508 Douai, France.

nor-edine.abriak@mines-douai.fr ; mahfoud.benzerzour@mines-douai.fr

\section{Résumé :}

La méthodologie d'étude de sédiments de dragage marins présentée dans cet article s'inscrit dans le cadre du projet de recherche Européen SETARMS cofinancé par l'Union Européenne (Interreg IVA). Ce projet européen a pour objectifs, entre autres, de mieux connaître les caractéristiques des sédiments marins en Manche et de mettre en place des solutions innovantes et durables de gestion des sédiments dragués en Manche (France-Angleterre) pour une application en techniques routières. Ces objectifs s’inscrivent dans les recommandations du groupe $\mathrm{n}^{\circ} 11$ "Sédiment de dragage" du Grenelle de la Mer (MINISTERE DE L’ENVIRONNEMENT, 2010).

Dans cet article, le projet d'ensemble est présenté tout en détaillant l'enchainement des études techniques dans le cadre méthodologique précisé. L’étape 1 de cette méthodologie consiste à caractériser les sédiments et à les classer d’un point de vue géotechnique et géochimique selon les réglementations et les guides en vigueur. Les réponses issues de l'étape 2 de traitement aux liants hydrauliques seront analysées afin de dégager des tendances et des corrélations entre les propriétés géotechniques et géochimiques d'un côté et l'aptitude au traitement aux liants hydrauliques et donc à la valorisation de l'autre. L'étape 3 consiste à choisir les sédiments éligibles aux formulations finales et aux tests in situ sur planches expérimentales.

La mise en œuvre de cette méthodologie, adaptée des guides techniques en vigueur, permettra de dégager des familles de sédiments à partir des sédiments étudiés dans le projet et soulève un certains nombre de questions et/ou de remarques.

Mots-clés :

Sédiments de dragage marins - Méthodologie - Acceptabilité environnementale Techniques routières - SETARMS - Valorisation 


\section{Introduction}

La France, avec un littoral (Métropole) de plus de 5800 km (SHOM, site web), drague chaque année en moyenne $50 \mathrm{Mm}^{3}$ de sédiments afin de maintenir l'exploitation de ses infrastructures portuaires. La Manche et la Mer du Nord constituant un enjeu économique, écologique et patrimonial majeur pour la France, plusieurs partenaires (universitaires et écoles d'ingénieurs, pouvoirs publics et collectivités, acteurs portuaires et industriels) se sont groupés au sein du projet SETARMS (Sustainable Environmental Treatment and Reuse of Marine Sediment). De par sa structure partenariale et ses objectifs, ce projet européen, permettra de trouver des solutions économiques et environnementales au dragage, basées sur des travaux scientifiques et techniques. En outre, le projet SETARMS, cofinancé par l'Union Européenne (Interreg IVA), s’inscrit dans les recommandations proposées par le groupe $n^{\circ} 11$ "Sédiment de dragage" du Grenelle de la Mer (Grenelle Mer, 2010) sur le devenir et la gestion des sédiments de dragage en terme d'acquisition des connaissances scientifiques et techniques sur les sédiments de dragage et la promotion des bonnes pratiques, des connaissances acquises et des retours d'expériences.

La revue des nombreux travaux de recherche en France sur le traitement et la valorisation des sédiments, montrent une étendue assez large des techniques et des méthodologies. Ainsi, selon l'objectif de la valorisation et le caractère immergeable ou non des sédiments, les méthodologies d'études diffèrent malgré des étapes similaires (COLIN, 2003 ; BOUTOUIL, 2006 ; DUBOIS, 2006 ; SEMCHA, 2006 ; GROSDEMANGE et al., 2008 ; AQUA et al., 2009 ; TRAN, 2009 ; ZRI et al., 2011).

S'intéressant plus particulièrement aux traitements aux liants hydrauliques et à la valorisation en technique routière (sous-couches ou remblais), le projet opérationnel SEDIMARD 83 s'est focalisé sur des traitements de sédiments par inertage. Les résultats de l'étude de valorisation des sédiments traités (SANNIER et al., 2009 ; LEVACHER et al., 2011) montrent que les performances mécaniques (seuil de $1 \mathrm{MPa}$ exigé pour la résistance à la compression simple à 28 jours) associées à une approche environnementale et économique sont atteintes avec plusieurs combinaisons de prétraitement et traitement.

Dans cet article, nous présenterons le projet SETARMS dans sa globalité puis la méthodologie pratique qui a été adoptée depuis le prélèvement des sédiments jusqu’à la valorisation des sédiments. Le projet étant en cours de réalisation, ne seront abordées que les phases terminées à la date de la rédaction de cet article.

\section{Présentation générale du projet SETARMS}

La gestion des matériaux de dragage générés par cette activité est confrontée à des difficultés juridiques, financières, environnementales, techniques et scientifiques. C'est dans ce contexte que le projet SETARMS (Sustainable Environmental Treatment and Reuse of Marine Sediment) a été proposé et sélectionné dans le cadre européen de 


\section{XII ${ }^{\text {èmes }}$ Journées Nationales Génie Côtier - Génie Civil \\ Cherbourg, 12-14 juin 2012}

coopération transfrontalière INTERREG IVA France (Manche)-Angleterre et cofinancé par le FEDER.

Le projet Européen SETARMS a pour objectifs de :

- Mieux connaître les caractéristiques des sédiments marins en Manche.

- Développer et promouvoir des pratiques durables de gestion des sédiments marins.

- Développer et fournir des supports et des outils de gestion transfrontalière.

- Créer un pôle d'excellence scientifique transfrontalier et des relations pérennes entre partenaires sur la Manche.

Afin de répondre aux objectifs fixés, le projet est structuré en 4 "Workpackages (WP)" comme le montre le tableau 1.

Tableau 1. Workpackages du projet SETARMS.

\begin{tabular}{|c|c|c|}
\hline Workpackage & Objectifs spécifiques & Coordinateur \\
\hline $\begin{array}{l}\text { WP } 1 \text { : Etat des lieux et perspectives } \\
\text { du dragage en Manche }\end{array}$ & $\begin{array}{l}\text { Etat des lieux et perspectives du dragage } \\
\text { en Manche, évaluation du potentiel du } \\
\text { travail en commun, méthodes de dragage } \\
\text { et harmonisation des pratiques }\end{array}$ & $A P L M$ \\
\hline WP 2 : Caractérisation des sédiments & $\begin{array}{l}\text { Etudes des caractéristiques de base des } \\
\text { sédiments avant et après traitement : } \\
\text { géotechnique, géochimique et } \\
\text { biodisponibilité }\end{array}$ & $U o B$ \\
\hline WP 3 : Valorisation des sédiments & $\begin{array}{l}\text { Etudes de valorisation des sédiments par } \\
\text { cimentation et études du potentiel des } \\
\text { méthodes électrocinétiques }\end{array}$ & ESITC Caen \\
\hline $\begin{array}{l}\text { WP } 4 \text { : Synthèse et diffusion des } \\
\text { résultats }\end{array}$ & $\begin{array}{l}\text { Travail de suivi, de communication et de } \\
\text { diffusion des résultats du projet }\end{array}$ & $A P L M$ \\
\hline
\end{tabular}

De par ses objectifs, le projet SETARMS, s'inscrit dans les recommandations proposées par le groupe $n^{\circ} 11$ "Sédiment de dragage" du Grenelle de la Mer (Grenelle Mer, 2010) sur le devenir et la gestion des sédiments de dragage en terme d'acquisition des connaissances scientifiques et techniques sur les sédiments de dragage et la promotion des bonnes pratiques, des connaissances acquises et des retours d'expériences.

Le partenariat regroupe plusieurs acteurs qui mettent leurs compétences en commun afin que les résultats du projet soient pragmatiques, innovants et applicables en Manche et au-delà. Ainsi, les partenaires du projet SETARMS sont :

- Laboratoires universitaires et écoles d'ingénieurs : ESITC Caen, Université de Caen Basse-Normandie (UCBN-ERPCB), LASEM, Ecole des Mines de Douai (EMD), Université de Brighton (UoB) et Université d'Exeter (UoE).

- Pouvoirs publics, collectivités : Conseil Général des Côtes d’Armor. 
- Acteurs portuaires : Association des Ports Locaux de la Manche (APLM, chef de file du projet) et Grand Port Maritime du Havre.

- Industriels : entreprise Eurovia Basse-Normandie.

\section{Méthodologie générale SETARMS}

La démarche générale adoptée ainsi que les actions prévues pour la réalisation du projet SETARMS sont illustrées par le schéma de la figure 1. Elle englobe l'ensemble des étapes qui seront suivies par l'ensemble des partenaires pour répondre aux objectifs du projet. Les étapes vont du prélèvement jusqu’à la valorisation. La valorisation retenue dans le projet SETARMS est la réutilisation des sédiments traités en techniques routières et plus précisément en couche de forme.

La figure 1 présente la méthodologie proposée dans le projet SETARMS. Elle fait apparaître le contenu de chaque étape de façon plus précise ainsi que les WP concernés. La démarche proposée repose sur un ensemble de réglementations et de guides en vigueur à ce jour et a pour objectif de dégager des familles de sédiments à partir des sédiments étudiés dans le projet. Ceux-ci sont tous sujets à l'étape 1 de caractérisations géotechnique et géochimique et de classifications ainsi qu'à un premier traitement aux liants hydrauliques tel que préconisé par le GTS.

Les réponses (étape 2) des sédiments étudiés en terme d'aptitude au traitement et de comportement mécanique et environnemental seront analysées afin de dégager des tendances et des corrélations entre les propriétés géotechniques et géochimiques d'un côté et l'aptitude au traitement aux liants hydrauliques et donc à la valorisation de l'autre. L'étape 3 consiste à choisir les sédiments éligibles aux formulations finales et aux tests in situ sur planches expérimentales.

\section{4. Échantillonnage et prélèvements}

Le projet SETARMS recouvre la caractérisation de 13 sédiments, 8 provenant de ports français, 5 de ports anglais. Les ports de prélèvement sont localisés sur la carte de la figure 2. Ces ports ont été sélectionnés dès le début du projet et l'échantillonnage a été réalisé entre mars 2010 et juin 2011. 


\section{XII ${ }^{\text {èmes }}$ Journées Nationales Génie Côtier - Génie Civil \\ Cherbourg, 12-14 juin 2012}

\section{Méthodologie SETARMS}

\begin{tabular}{|c|c|c|c|c|}
\hline$\checkmark$ Prélèvement & Sed. A & Sed. B & Sed. ... & Sed. X \\
\hline $\begin{array}{l}\checkmark \text { Caractérisation } \\
\text { géochimique (WP2) } \\
\checkmark \text { Caractérisation } \\
\text { géotechnique (WP3) }\end{array}$ & \multicolumn{4}{|c|}{$\begin{array}{l}1^{\text {et }} \text { niveau de classification et base de données sur les sédiments } \\
\text { étudiés: } \\
>\text { Niveaux GEODE (FR), CEFAS (UK) (WP1 et WP2) } \\
>\text { Circulaire } 4 \text { juillet 2008 (WP1 et WP2) } \\
>\text { Guides GTR, GTS (WP3) } \\
>\text { Guide méthodologique Ministère Nov. 2010... (WP1, WP2 et WP3) }\end{array}$} \\
\hline $\begin{array}{l}\checkmark \text { Analyses résultats } \\
\text { étape } 1 \\
\checkmark \text { Traitements et } \\
\text { formulations (WP3) } \\
\checkmark \text { Analyses } \\
\text { géochimiques (WP2) }\end{array}$ & $\begin{array}{l}>\text { Prétrai } \\
\text { de la chat } \\
>\text { Compo } \\
>\text { Impact } \\
>\text { Analyse }\end{array}$ & $\begin{array}{l}\text { ulation : } \\
\text { criblage 0/ } \\
\text { roctor, te: } \\
\text { mécaniqu } \\
\text { lemental( } \\
\text { mentaires }\end{array}$ & $\begin{array}{l}+6 \% \text { CEI } \\
\text { hydratatio } \\
\text { au traitem } \\
\text { forme, GT } \\
\text { géotechn }\end{array}$ & $\begin{array}{l}\text { le fixation } \\
\text { 3) } \\
\text { et WP3) }\end{array}$ \\
\hline $\begin{array}{l}\checkmark \text { Analyses résultats } \\
\text { étape } 2 \\
\checkmark \text { Sélection et choix } \\
\text { sédim/formulations }\end{array}$ & $\begin{array}{l}>\text { Sédim } \\
>\text { Prétra } \\
>\text { Formı } \\
>\text { Prépa }\end{array}$ & $\begin{array}{l}\text { mélange } \\
\text { adapté } \\
\text { daptée } 6 \\
\text { es chanti }\end{array}$ & $\begin{array}{l}\text { ts éligible: } \\
\text { n au labor } \\
\text { : route ex }\end{array}$ & \\
\hline
\end{tabular}

Figure 1. Méthodologie SETARMS et interaction entre les WP (BOUTOUIL et al., 2011).

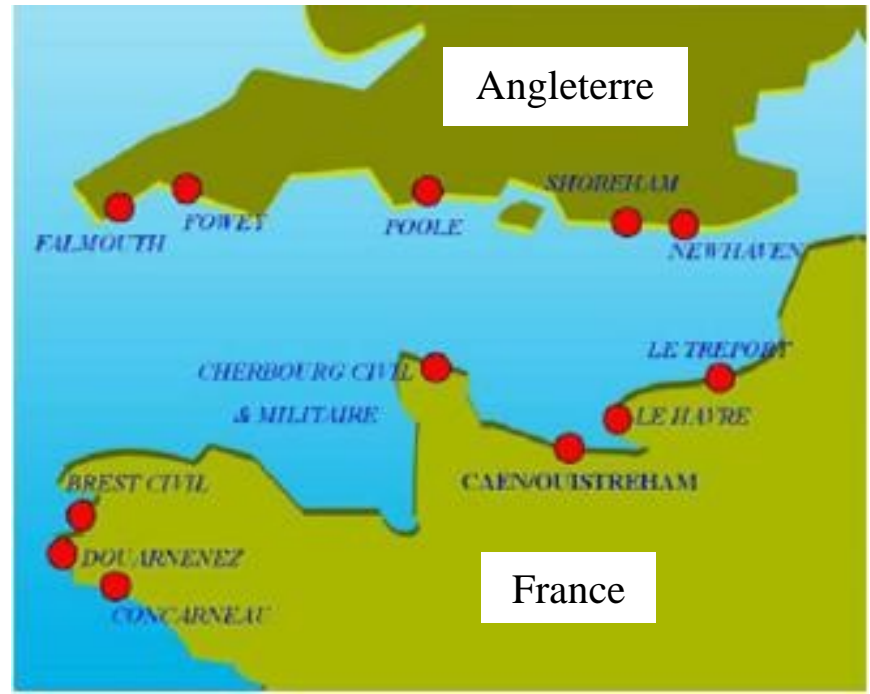

Figure 2. Localisation des prélèvements.

L'un des problèmes majeurs rencontrés est le transfert des échantillons de sédiments entre les deux pays, France et Angleterre, le transport de grandes quantités de sédiments 
pour une caractérisation géotechnique posant problème en terme de formalités douanières. Ainsi, l'ESITC Caen n'a pas reçu d'échantillons anglais à analyser tout comme les universités anglaises (Brighton et Exeter) n’ont reçu aucun échantillon français pour la caractérisation géotechnique. Les échanges de sédiments se sont réalisés à de faibles quantités permettant les analyses chimiques de part et d'autre de la Manche. Par ailleurs, afin de garantir la confidentialité des données relatives à chaque port et la traçabilité des sédiments, chacun est codé, par une lettre d'abord, identifiant le port de prélèvement, puis par un numéro relatif au nombre de prélèvement. Ces codes sont utilisés tout au long du projet. Ainsi chaque port a accès seulement à ses propres données et les scientifiques ont accès aux informations dont ils ont besoins pour la traçabilité des données.

Le suivi des prélèvements est effectué grâce à une fiche type de prélèvement remplie (date, point GPS, profondeur par rapport au fond marin, etc.) par les autorités portuaires.

Les prélèvements sont effectués selon les moyens des ports. Le tableau 2 résume les principales caractéristiques de prélèvement des sédiments. Les sédiments sont identifiés par leur code.

Tableau 2. Paramètres de prélèvement.

\begin{tabular}{lllll}
\hline Identification & $\begin{array}{l}\text { Profondeur } \\
(\boldsymbol{m})\end{array}$ & Mode & $\begin{array}{l}\text { Teneur en eau (\%) } \\
\text { Moy. }(\text { min-max) }\end{array}$ & $\begin{array}{l}\text { Concentration solide } \\
\text { moyenne }\left(\boldsymbol{g} . \mathbf{L}^{-1}\right)\end{array}$ \\
\hline A001 & 7 & Benne & $115(115-116)$ & 470 \\
B001 & 8 & Plongeurs & $70(66-73)$ & 590 \\
C001 & $/$ & Plongeurs & $230(170-333)$ & 300 \\
D001 & 13 & Drague & $99(93-104)$ & 500 \\
E001 & $/$ & Benne & $188(167-210)$ & 350 \\
F001 & 6 & Plongeurs & $235(233-236)$ & 300 \\
G001 & 15 & Plongeurs & $97(76-109)$ & 510 \\
M001 & $/$ & $/$ & $200(97-202)$ & $/$ \\
\hline
\end{tabular}

L'un des critères d'échantillonnage évoqué précédemment est la représentativité du prélèvement. Il est ainsi nécessaire de faire état des pratiques habituelles des ports. Le WP 1 donne quelques informations générales sur les protocoles de dragage. Les moyens utilisés sont similaires pour la France et l'Angleterre. Ils sont majoritairement mixte (hydraulique et mécanique combiné). Les fréquences de dragage sont la plupart du temps annuelles (50\%). Dans 33\% des cas une durée supérieure à 5 ans entre deux opérations de dragage est observée pour la France. L’immersion est la destination majoritairement utilisée en France et en Angleterre (plus de 95\%). Les sites d'immersion sont majoritairement compris entre 1 et $3 \mathrm{MN}$ en France, quand ils sont 


\section{XII ${ }^{\text {èmes }}$ Journées Nationales Génie Côtier - Génie Civil \\ Cherbourg, 12-14 juin 2012}

situés à plus de $3 \mathrm{MN}$ en Angleterre. Aucune filière terrestre n'est commercialement viable à l'heure actuelle en France comme en Angleterre.

\section{Conclusions}

Dans cet article, le projet SETARMS a été présenté avec ses objectifs, ses partenaires et sa méthodologie. L'étape de prélèvement dans les 13 ports du projet a été décrite dans le cadre d'un protocole qui a été défini avec l'ensemble des partenaires. Les étapes de prélèvement et de caractérisation géotechnique et géochimique constituent des étapes cruciales. Elles ont consisté non seulement à réaliser des essais de laboratoire mais ont permis également de soulever un certain nombre de questions et de problèmes, résolus au cours du projet, et liés à :

- L'application de différents protocoles par les laboratoires partenaires du projet.

- La classification des sédiments qui dépend de l'option de gestion de ces sédiments : dépôt à terre ou immersion en mer.

- L'utilisation de règlements et de normes provenant de différents domaines qui vont de la classification des sédiments jusqu'au guide de traitement des sols, en passant par les normes de lixiviation et la mesure des teneurs de certaines substances chimiques particulières.

Les résultats détaillés de caractérisation géotechniques et géochimiques ainsi que ceux relatifs aux traitements et formulation pour une utilisation en sous-couche routière sont disponibles dans les rapports techniques des différents workpackages et des articles en cours de publication.

\section{Remerciements}

Les résultats de cet article sont issus du projet européen SETARMS (Interreg IV-A, France-manche). Les auteurs remercient l'Union Européenne et le Conseil Régional de Basse Normandie, cofinanceurs de SETARMS.

\section{Références bibliographiques}

AQUA J.-L., BOISSERY P., ALZIEU C. (2009). Un programme opérationnel d'identification des modes de gestion durable des sédiments marins : le projet SEDI.MAR.D. 83. Revue Paralia, n 2, pp s1.1-s1.12. doi:10.5150/revue-paralia.2009.s01

BOUTOUIL M, BEN ABDELGHANI F, SAUSSAYE L, MAHERZI W, (2011). Caractérisation géotechnique des sédiments bruts et introduction à l'électrocinétique. Rapport Technique 2, WP3, projet SETARMS, Octobre 2011, 44 p.

BOUTOUIL M. (2006). Contributions à la connaissance du comportement mécanique et environnemental des sédiments et sols solidifiés/stabilisés. Mémoire d'Habilitation à Diriger des Recherches, Université de Caen, 54 p.

COLIN D. (2003). Valorisation des sédiments fins de dragage en technique routière. Thèse, Université de Caen. 
DUBOIS V. (2006). Étude du comportement physico-mécanique et caractérisation environnementale des sédiments marins. Thèse de l'Université d'Artois.

GROSDEMANGE D., LEVEQUE F., DROUSIE J.L., AQUA J.L., MEHU J., BAZIN C. (2008). The SEDIMARD project: Presentation and results. I2SM, Lille 9-11 juillet 2008, pp 181-186.

LEVACHER D., SANCHEZ M., DUAN Z., LIANG Y. (2011). Valorisation en unité pilote de sédiments méditerranéens : étude des caractéristiques géotechniques et de la perméabilité. Revue Paralia, Vol. 4, pp 4.1-4.20. doi:10.5150/revue-paralia.2011.004 MINISTERE DE L'ENVIRONNEMENT (2010), Sédiment de dragage, rapport final du groupe de travail $\mathrm{n}^{\circ} 11$. Direction de l'Eau et de la Biodiversité, $35 \mathrm{p}$.

SANNIER L., LEVACHER D., JOURDAN M. (2009). Approche économique et validation de méthodes de traitements aux liants hydrauliques de sédiments marins contaminés. Revue Paralia, $\mathrm{n}^{\circ}$ 2, pp. s2.1-s2.15. doi:10.5150/revue-paralia.2009.s02

SEMCHA A. (2006). Valorisation des sédiments de dragage: Applications dans le BTP, cas du barrage de Fergoug. Thèse, université de Reims.

SHOM (site web). http://www.shom.fr/

TRAN N. T. (2009), Valorisation de sédiments marins et fluviaux en technique routière, Thèse de l'Université d'Artois, 225 p.

ZRI A., ABRIAK N.E., ZENTAR R. (2011). Caractérisation physico-mécanique d'un sédiment brut et traité à la chaux. EJECE, Vol. 15, n²/2011, pp. 239-267. 